\title{
Catalytic Synthesis of Fatty Acid Esters of Isethionate with High Surface Activity
}

\author{
Gongxiao Zhang \\ Department of Chemical Engineering, \\ Taishan Medical University, \\ Taian, 271016, China \\ e-mail: gxzhang@tsmc.edu.cn
}

\author{
Long Zhang \\ School of Chemical Engineering, \\ Changchun University of Technology, \\ Changchun, 130012, China \\ e-mail: zhanglongzhl@163.com
}

\begin{abstract}
In this report, a kind of fatty acid esters of isethionate was synthesized with myristic acid and sodium hydroxyethyl sulfonate as raw materials and methane sulfonic acid as catalyst. Then, the effects of molar ratio of reactants, reaction temperature, catalyst dosage and reaction time on the product yield were discussed. The optimal reaction conditions are determined as follows: the molar ratio of myristic acid to sodium hydroxyethyl sulfonate was 1.2 , the reaction temperature was $160^{\circ} \mathrm{C}$, the weight ratio of methane sulfonic acid to myristic acid was $2.5 \%$, the reaction time was 4 hours, the obtained product yield was $68.7 \%$. Finally, the surface activity of as-obtained product was evaluated by the surface tension $(28.4 \mathrm{mN} / \mathrm{m})$ and the critical micelle concentration $\left(1.21 \times 10^{-3} \mathrm{~mol} / \mathrm{L}\right)$, indicating that the product is a fine surfactant.
\end{abstract}

Keywords-fatty acid; esters of isethionate; myristic acid; surface activity; catalytic synthesis

\section{INTRODUCTION}

Detergent, whose output value on the market amounted to $\$ 60$ billion for global commercial cleaning products, has been an important product in the surfactant industry [1]. Among them, laundry cleaning products account for the largest percentage. The low-cost anionic surfactants are the main raw material of soap and other cleaning products. The alkaline fatty acid salt [2], a dominant product twenty centuries ago, has been gradually replaced by new surfactants, such as linear alkyl benzene sulfonate (LAS) [3] for its shortages.

Fatty acid esters of isethionate $\left(\mathrm{RCOOCH}_{2} \mathrm{CH}_{2} \mathrm{SO}_{3} \mathrm{M}, \mathrm{R}\right.$ is 9-17, $\mathrm{M}$ is $\mathrm{Na}$ or $\mathrm{NH}_{4}$ etc.), a kind of fatty acid-derived anionic surfactants, has good surface activity and biodegradability. It has been widely used in daily chemicals and other industrial areas and has also become a main anionic surfactant product.

Currently, the synthetic methods of the fatty acid esters of isethionates include the esterification with acid chloride as raw materials and trans-esterification method [4]. A big drawback of the first method, with acid chloride as raw materials, is the high cost of the raw materials. Besides, the waste treatment is also another obstacle [5]. The second method, with low-cost fatty acid as raw materials, needs a higher reaction temperature without environmental pollution.

In this report, a novel kind of fatty acid esters of isethionate was synthesized with myristic acid and sodium hydroxyethyl sulfonate as raw materials and methane sulfonic acid as catalyst. The optimum conditions were discussed. Finally, two parameters (the surface tension, critical micelle concentration) were also measured to evaluate its surface activity. The results showed that the assynthesized product has excellent surface activity.

\section{EXPERIMENTAL}

Myristic acid, technical grade, Shanghai Zongzhou Chemical Co., Ltd.; Sodium hydroxyethyl sulfonate, technical grade, Jilin Zhongxin Chemical Co., Ltd.; Methane sulfonic acid, analytical reagent, $98 \mathrm{Wt} \%$, Aladdin Chemical Co., Ltd. .

Nicolet IS10 Fourier Infrared spectrometer (Shimadzu Seisakusho); Bruce AVANCEIII NMR (400 MHz, Bruce Company); X-4 digital display micro melting point apparatus (Beijing Tektronix Instrument Co.). K12 full-automated surface tension instrument (Germany KrUss Instrument Co.).

In a reactor, equipped with temperature control apparatus, condensing apparatus, stirring apparatus and nitrogen protection apparatus, certain amount of myristic acid and methane sulfonic acid were added and heated until it is melted completely. Then, a certain amount of sodium hydroxyethyl sulfonate aqueous solution was added. After a period of reaction time at a given temperature, then it was cooled into room temperature, the residual water removed continuously. After the completion of the reaction, a certain amount of anhydrous ethanol was added into the reactor, and stirred until a suspension was formed. The product was separated and purified with repeating the above processes for three times.

\section{RESULTS AND DISCUSSIONS}

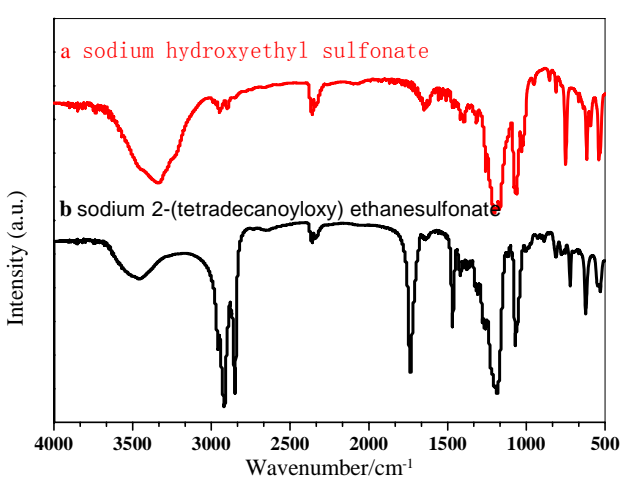

Figure 1. FT-IR spectra of the product. 
The FT- IR spectrum of the product was shown in Figure 1. As can be seen from Figure 1,the $-\mathrm{C}=\mathrm{O}$ absorption peak at $1734 \mathrm{~cm}^{-1}$ appears, while strong absorption peak at 1186 $\mathrm{cm}^{-1}$ also appears. It indicates the presence of the ester groups $[6,7]$, suggesting that the desired product was synthesized successfully. Sub-headings should be typeset in boldface italic and capitalize the first letter of the first word only.

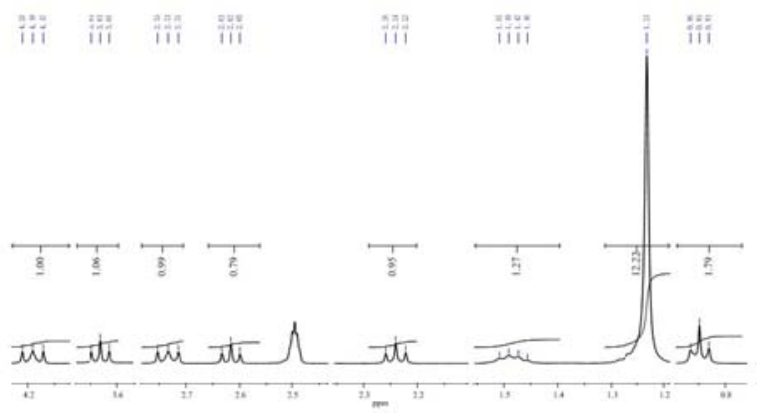

Figure 2. $\mathrm{H}^{1} \mathrm{NMR}$ spectrum of the product

The $\mathrm{H}^{1}$ NMR spectrum of the product was shown in Figure 2. The peak at 4.19 corresponds to sulfonate ester group with a single bond connected to a methylene carbon and oxygen. The peaks around 3.63, 2.73, 2.62, 2.24, 1.48, 1.23 and 0.85 are assigned to the sulfonate group attached to the methylene hydrogen, methylene hydrogen directly connected with the carbonyl group, and the interval with a carbonyl methylene $\mathrm{CH}_{2}$ hydrogen $\left(\mathrm{COOCH}_{2} \mathrm{CH}_{2}\right)$, the interval between two carbonyl $\mathrm{CH}_{2}$ methylene hydrogens $\left(\mathrm{COOCH}_{2} \mathrm{CH}_{2} \mathrm{CH}_{2}\right)$, the carbonyl interval three $\mathrm{CH} 2$ methylene hydrogens $\left(\mathrm{COOCH}_{2} \mathrm{CH}_{2} \mathrm{CH}_{2} \mathrm{CH}_{2}\right)$ and the remaining nine methylene hydrogen, and three methyl hydrogen, respectively.

Orthogonal test was employed to obtain the optimal reaction condition. Molar ratio of reactants, reaction temperature, catalyst dosage and reaction time were chosen as the main factors. The four factors and three levels orthogonal table was shown in Table I, the yield of the desired product was chosen as an index y (Table II).

TABLE I. FOUR FACTORS AND THREE LEVELS ORTHOGONAL DESIGN TABLE

\begin{tabular}{clccc}
\hline \multirow{2}{*}{$\begin{array}{c}\text { Level } \\
\text { s }\end{array}$} & $\begin{array}{l}\text { Reactant } \\
\text { Mole } \text { Ratio } \\
\text { (A) }\end{array}$ & $\begin{array}{l}\text { Reaction } \\
\text { Time }\end{array}$ & $\begin{array}{c}\text { Reaction } \\
\text { Temperature } \\
(\text { (C)/oC }\end{array}$ & $\begin{array}{l}\text { Catalyst } \\
\text { Dosage } \\
(\boldsymbol{D}) / \%\end{array}$ \\
\hline 1 & $1: 1.0$ & 2.5 & 160 & 2.5 \\
2 & $1.2: 1$ & 3.0 & 180 & 5.0 \\
3 & $1.5: 1$ & 4.0 & 200 & 7.5 \\
\hline
\end{tabular}

As can be seen from Table II, the reaction time (B) has the greatest effect on the yield, following by the reaction temperature $(\mathrm{C})$. With the increasing of the reaction time, the esterification carried out continuously with the increasing conversion. A higher reaction temperature is beneficial for the esterification .If the reaction temperature is too high $(\geqslant$ $200{ }^{\circ} \mathrm{C}$ ), the color of the product became deeper, from white to pale yellow. The amount of catalyst (D) has little effect on the reaction. The optimal reaction conditions were A2B3C1D1. That is: the molar ratio of myristic acid and sodium hydroxyethyl sulfonate was 1.2 , the reaction temperature was $160^{\circ} \mathrm{C}$, the weight ratio of methane sulfonic acid to myristic acid was 2.5 , the reaction time was 4 hours and the product yield was $68.7 \%$.

TABLE II. The DATA OF ORTHOgOnALTEST AND RANGE ANALysis

\begin{tabular}{llllll}
\hline Samples & $\mathbf{1 ( A )}$ & $\mathbf{2 ( B )}$ & $\mathbf{3 ( C )}$ & $\mathbf{4 ( D )}$ & $\mathbf{Y} / \%$ \\
\hline 1 & 1 & 1 & 1 & 1 & 60.2 \\
2 & 1 & 2 & 2 & 2 & 61.1 \\
3 & 1 & 3 & 3 & 3 & 63.1 \\
4 & 2 & 1 & 2 & 3 & 61.2 \\
5 & 2 & 2 & 3 & 1 & 64.8 \\
6 & 2 & 3 & 1 & 2 & 68.7 \\
7 & 3 & 1 & 3 & 2 & 57.7 \\
8 & 3 & 2 & 1 & 3 & 67.3 \\
9 & 3 & 3 & 2 & 1 & 68.3 \\
k1 & 61.497 & 59.697 & 65.413 & 64.437 & \\
k2 & 64.893 & 64.407 & 63.523 & 62.507 & \\
k3 & 64.413 & 66.700 & 61.867 & 63.860 & \\
Range & 3.396 & 7.003 & 3.546 & 1.930 & \\
Sequence & B>C>A>D & & & \\
Optimal Level & A2 & B3 & C1 & D1 & \\
Optimal & A2B3C1D1 & & & \\
Assembly & & & &
\end{tabular}

Under the optimum reaction conditions, the surface activity of the product was evaluated. The surface tension with the concentration variation curve was shown in Figure 3. The measured surface tension was $28.4 \mathrm{mN} / \mathrm{m}$ and the critical micelle concentration was $1.21 \times 10^{-3} \mathrm{~mol} / \mathrm{L}$. Therefore, the as-obtained product has a low surface tension and critical micelle concentration, indicating its good surface activity.

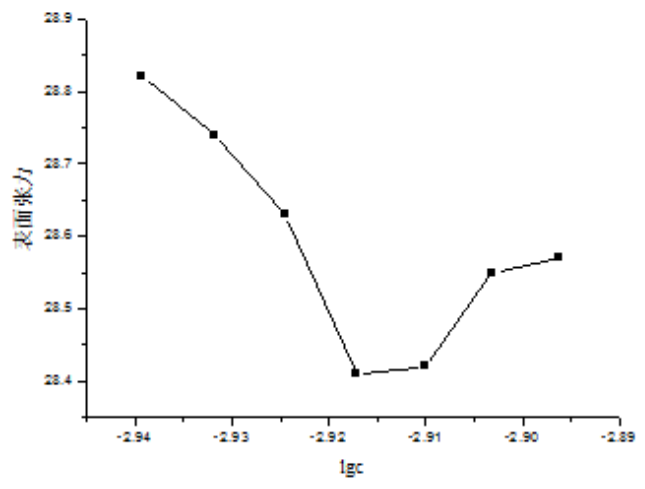

Figure 3. The relationship between concentration and interfacial tension 


\section{CONCLUSIONS}

A kind of fatty acid esters of isethionate was synthesized with myristic acid and sodium hydroxyethyl sulfonate as raw materials and methane sulfonic acid as catalyst. The optimum reaction conditions are determined by orthogonal experiment design. The surface activity of as-obtained product was evaluated by the surface tension $(28.4 \mathrm{mN} / \mathrm{m})$ and the critical micelle concentration $\left(1.21 \times 10^{-3} \mathrm{~mol} / \mathrm{L}\right)$, indicating that the product is a fine surfactant. The synthetic process is of mild reaction conditions without environmental pollution. This novel product is expected to be applied for the preparation of micro-emulsion diesel.

\section{REFERENCES}

[1] J. J. Scheibel, J Surfactants Deterg, vol. 17, 2004, pp. 319-328.

[2] D.Y. Zhang, Surfact. Soap Deterg. Ind. China, vol. 14, 2001, pp. 5157

[3] Y.X. Yu, J. Zhao and A. E. Bayly, Chinese J. Chem. Eng, vol. 16, 2008, pp. 517-527.

[4] B.C. Xu. H.Y. Nie, and Z.Q. Qu, Mod. Chem. Ind, vol. 28, 2008, pp. 366-368.

[5] G.X. Zhang, Y.B. Qi. H.B. Zhang. J. Wuhan Univ. Technol, vol. 1, 2013, pp. 44-47.

[6] P.Y. Wang. H. Hu. Fine Chem, vol. 21, 2004, pp. 906-908.

[7] N. Cao, Chinese J. Spectrosc. Lab, vol. 24, 2007, pp. 1099-1101. 